\title{
How is diversity related to species turnover through time?
}

\author{
Jonathan B. Shurin \\ J. B. Shurin(shurin@zoology.ubc.ca),Dept of Zoology, Univ. of British Columbia, 6270 Univ. Blvd., Vancouver, Canada, BC V6T \\ $1 Z 4$.
}

\begin{abstract}
Empirical studies across a wide range of taxa show that the slopes of species-time relationships often decline as average species richness increases, indicating that more diverse communities have greater temporal stability in species composition. I explored potential explanations for this observation using two simple model formulations for species temporal dynamics. In the Abiotic model, species turnover is governed by the degree of heterogeneity in the environment and the range of species' tolerances. In this case, more variable conditions lead to lower species richness and higher turnover, but only if the distribution of species' niche widths and the size of the species pool are independent of the degree of environmental variability. The Biotic model represents direct effects of diversity on turnover through positive or negative feedbacks between diversity and species' colonization and extinction rates. Declining turnover with increasing richness occurred when higher diversity either facilitated colonization by new species or reduced extinction rates of extant species. Both models could produce the observed pattern of declining turnover at higher diversity under some circumstances, however the conditions for this outcome in the Abiotic model were restrictive and potentially unrealistic. The models provide a processbased framework for understanding the connection between diversity and species turnover through time.
\end{abstract}

Increases in the number of species encountered with either the area or time period of sampling are two of the most consistent patterns in ecology. Drawing biological inferences from variation in species-area or speciestime functions requires that the effects of sampling artifacts are separated from those of biological processes (Gotelli and Colwell 2001). Species-area relations have been used to make comparisons about the relative dispersal abilities of different taxonomic groups (Green et al. 2004, Hillebrand 2004, Horner-Devine et al. 2004), geographic and latitudinal variation in spatial turnover in species composition (Koleff et al. 2003, Hillebrand 2004), the self-similarity or scale-dependence of species distributions (Crawley and Harral 2001) and the response of diversity to anthropogenic or natural habitat loss (Seabloom et al. 2002, Schoener and Spiller 2006).

Although the mechanistic basis for systematic variation in the shape of the species-area relationship has been explored (Preston 1960, Connor and McCoy 1979, Sugihara 1980, He and Legendre 1996, 2002, Harte et al. 1999, Hubbell 2001), temporal turnover in species composition has received less theoretical attention. Variation in species composition through time arises out of a combination of random sampling errors that cause species to be detected at some times but not others (White 2004), colonization from neighboring habitats or dormant pools, and local extinction. More variable environments may show greater compositional turnover through time as species with different affinities are favored as prevailing conditions change (Chesson and Huntly 1997). Colonization and extinction are affected by ecological processes that influence average population sizes and their variability through time. For instance, smaller and more variable populations are at the highest risk of extinction (Pimm et al. 1988). Colonization is affected by a range of factors such as habitat size and isolation (MacArthur and Wilson 1967), system productivity (Davis et al. 2000), disturbance (Burke and Grime 1996, Shurin 2000), and native diversity (Levine and D'Antonio 1999). A mechanistic understanding of species-time patterns in terms of colonization and extinction rates will allow us to make predictions about how ecological factors that 
affect population growth and variability manifest themselves in patterns of species turnover.

Species-time relations show two consistent empirical regularities across a wide range of taxa. First, the spatial and temporal scales of sampling have interactive effects on the number of species encountered. The exponent of the species-time curve is smaller in larger areas, and the exponent of the species-area curve decreases when habitats are sampled over longer periods (Adler and Lauenroth 2003, Adler et al. 2005). Second, the rate of species accumulation through time is often lower when average community diversity is greater. White et al. (2006) show that the exponent of the species-time relationship declines as mean species richness increases in diverse groups of taxa including birds, mammals, plants and invertebrates. The second pattern indicates that species-time curves tend to converge at long temporal scales. Shurin et al. (2007) found that temporal turnover among zooplankton species declines with long-term diversity among lakes in the temperate zone, but that diversity and turnover are both lower at high latitude. Their results indicate that diversity and compositional stability are positively correlated within a biogeographic region, but negatively correlated over latitudinal gradients. White et al. (2006) concluded that "large temporal fluctuations in the environment might cause species to be more temporally patchy in their occurrence, which should lower species richness and increases temporal turnover". However, temporal variability can also enhance species coexistence through the storage effect, allowing species to persist by changing patterns of species dominance (Chesson and Warner 1981). In addition, species diversity per se may affect the temporal stability of populations. Diversity may either reduce (May 1974, Ives and Hughes 2002) or enhance (McCann et al. 1998) population stability depending on the distribution of interaction strengths among community members. If diversity affects colonization and extinction rates via effects on population stability, then the pattern of higher turnover at low diversity may be driven by species interactions rather than abiotic conditions.

I explored the connection between diversity and species turnover through time in simple heuristic models where colonization and extinction are driven either by temporal variability in the abiotic environment, or by emergent effects of diversity on colonization and extinction. In the Abiotic model, species' niches are distributed along a one-dimensional axis of environmental conditions that vary through time. Species persist when the environmental conditions fall within the range of their tolerance, otherwise they go extinct. In the Biotic model, species colonization and extinction rates depend on the number of co-occurring taxa. I modeled situations where diversity either enhances or decreases extinction and colonization and asked what effects increased diversity has on the rate of turnover under each scenario. The goal of the two models is to provide a process-based interpretation of patterns of species turnover through time. In particular, the models allow us to understand what mechanisms can produce the observed correlations between diversity and the exponent of the species-time relationship (White et al. 2006, Shurin et al. 2007).

\section{Models}

\section{Abiotic model}

The model of abiotic control of turnover is based on the idea that temporal environmental heterogeneity drives changes in community composition (Shmida and Wilson 1985). The model assumes that species are present whenever conditions fall within the bounds of their environmental tolerance, otherwise they go extinct. This is equivalent to assuming no dispersal limitation. Such "rescue effects" may occur through dormant stages capable of persisting through harsh periods in taxa like plants or aquatic micro-organisms, and in other organisms through dispersal from neighboring habitat patches (Hanski et al. 1996).

The model represents a single axis of abiotic variability with some range $\mathrm{W}_{\mathrm{H}}$ (habitat width, our measure of environmental heterogeneity). Each species i has a niche width $\mathrm{W}_{\mathrm{N}, \mathrm{i}}$ that is the range of condition over which it has positive population growth (assuming that all species tolerances fall within the range of $\mathrm{W}_{\mathrm{H}}$ ). If environmental conditions are drawn from a uniform random distribution at each time step, then the probability that each species is present at any moment in time $\left(p_{i}\right)$ is $W_{N, i} / W_{H}$. If $W_{N}$ is the same across species, then the average number of species present at any given time is $S_{\max } \times W_{N} / W_{H}$ where $S_{\max }$ is the number of species in the long-term species pool. The probability that a given species $\mathrm{i}$ has not occurred over some time interval $\mathrm{T}$ is $\mathrm{p}_{\mathrm{a}, \mathrm{T}}=\left(1-\mathrm{p}_{\mathrm{i}}\right)^{\mathrm{T}}$, therefore the likelihood of occurrence as a function of time is $1-\mathrm{p}_{\mathrm{a}, \mathrm{T}}$ and the cumulative number of species encountered by time $\mathrm{T}$ is

$\mathrm{S}_{\mathrm{T}}=\mathrm{S}_{\max }\left(1-\left(1-\mathrm{W}_{\mathrm{N}} / \mathrm{W}_{\mathrm{H}}\right)^{\mathrm{T}}\right)$

This expression illustrates the dependence of the speciestime curve on the number of species in the pool $\left(S_{\max }\right)$, the breadth of their environmental tolerance $\left(\mathrm{W}_{\mathrm{N}}\right)$, and the range of variability in the environment $\left(\mathrm{W}_{\mathrm{H}}\right)$. The key assumptions are that species have identical niche widths, that environmental variability is uniformly distributed and temporally uncorrelated, and that species are not colonization limited and go extinct immediately when conditions become unfavorable. I explored the consequences of relaxing some of these assumptions in Appendix 1. Figure 1 is a schematic diagram of the 


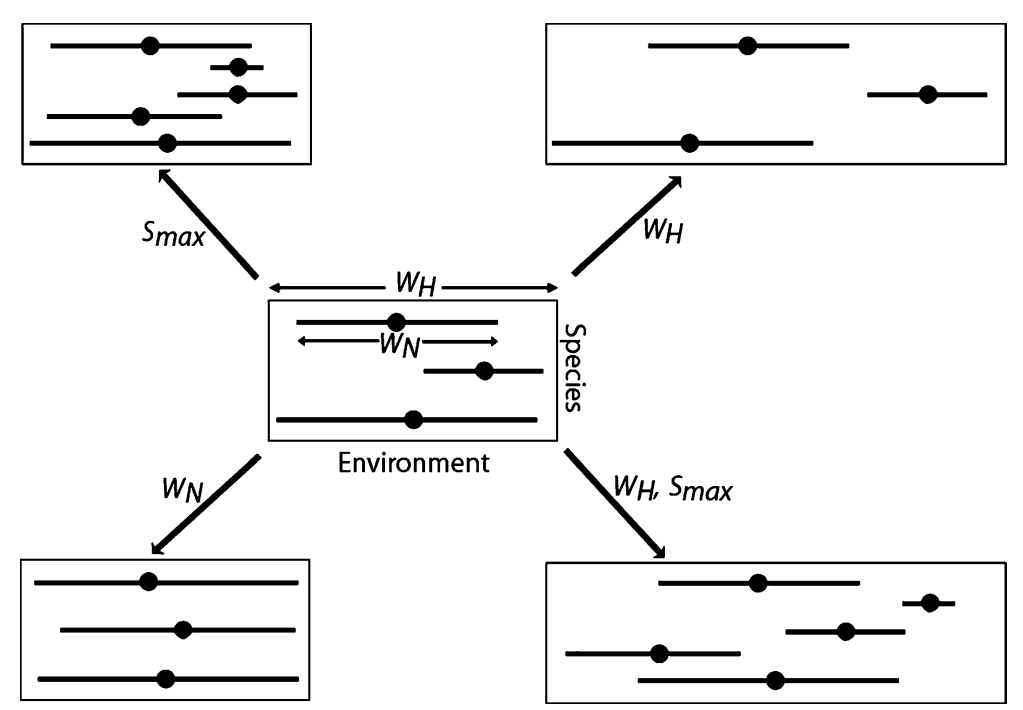

Fig. 1. A schematic diagram of the Abiotic model. The width of the box represents the range of environmental heterogeneity in the habitat $\left(\mathrm{W}_{\mathrm{H}}\right)$ and each line is the conditions where a species has positive growth $\left(\mathrm{W}_{\mathrm{N}}\right)$. Each arrow illustrates the effect of changing the indicated parameter without altering the others. The upper left shows increases in the size of the long-term species pool $\left(S_{\max }\right)$, the upper right is an increase in habitat variability $\left(W_{H}\right)$, the lower left is the species' niche widths $\left(W_{N}\right)$, and the lower right is when $\mathrm{W}_{\mathrm{H}}$ and $\mathrm{S}_{\max }$ increase in parallel.

effects of each parameter on the distribution of species' niches along the environmental axis.

Figure 2 shows the effects of the parameters in the Abiotic model on the species-time curves. Increasing $S_{\max }$ leads to species accumulation curves that diverge initially, reach asymptotes after similar time periods, and are parallel on log scales (Fig. 2A). Increasing environmental variability $\left(\mathrm{W}_{\mathrm{H}}\right)$ leads to lower average diversity, slower initial accumulation of new species, and species-time curves that increase for longer periods (Fig. 2B). Species' niche width $\left(\mathrm{W}_{\mathrm{N}}\right)$ has the opposite effect as $\mathrm{W}_{\mathrm{H}}$ (Fig. 2C) since the species-time curve depends on the ratio between the two (Eq. 1). The main result is that greater temporal heterogeneity $\left(\mathrm{W}_{\mathrm{H}}\right)$ without commensurate changes in the size of the species pool leads to lower average species diversity and greater turnover through time (steeper speciestime curves) as proposed by White et al. (2006). However, under this scenario, the density of species niches on the environmental axis declines with increasing variability (Fig. 1). If species' niche distributions are fixed on the environmental axis $\left(S_{\max } / W_{H}\right.$ is a constant), then the size of the long-term species pool increases with habitat breadth. That is, a more variable environment intersects with the niches of a greater number of species, increasing $S_{\max }$. If species' niche distributions are constant and independent of the environment, then the species-time curves diverge at long temporal scales (Fig. 2D). (The curves do not converge if we extend the plots to longer time scales.) In this case, a more variable environment allows more species to persist, increasing diversity and causing greater compositional turnover through time.

\section{Biotic model}

The Abiotic model assumes that species appearances and disappearances are driven by environmental fluctuations but independent of the identities or number of cooccurring species in the community. However, diversity may be regulated through positive or negative feedbacks driven by effects on colonization and extinction (Brown et al. 2001). Population and community stability are likely to be related if species that show more extreme density fluctuations are at greater risk of extinction (Pimm et al. 1988). Small populations are difficult to detect and may show apparent extinctions as a result of sampling errors. In addition, local populations may become extinct but be rescued by either neighboring habitats or dormant individuals (Chesson and Warner 1981, Hanski et al. 1996). Apparent colonization occurs when species rise from densities below detection limits, either from cryptic remnant populations, a dormant pool, or propagules from neighboring habitats. Extinction occurs when species decline below detection but may still be rescued by one of the above sources. The Biotic model represents communities where all individuals cannot be completely sampled, a situation that applies to nearly all natural communities. The frequency of appearance and disappearance of population is a measure of communitywide compositional stability. If species diversity affects the average population size or temporal variability, then 

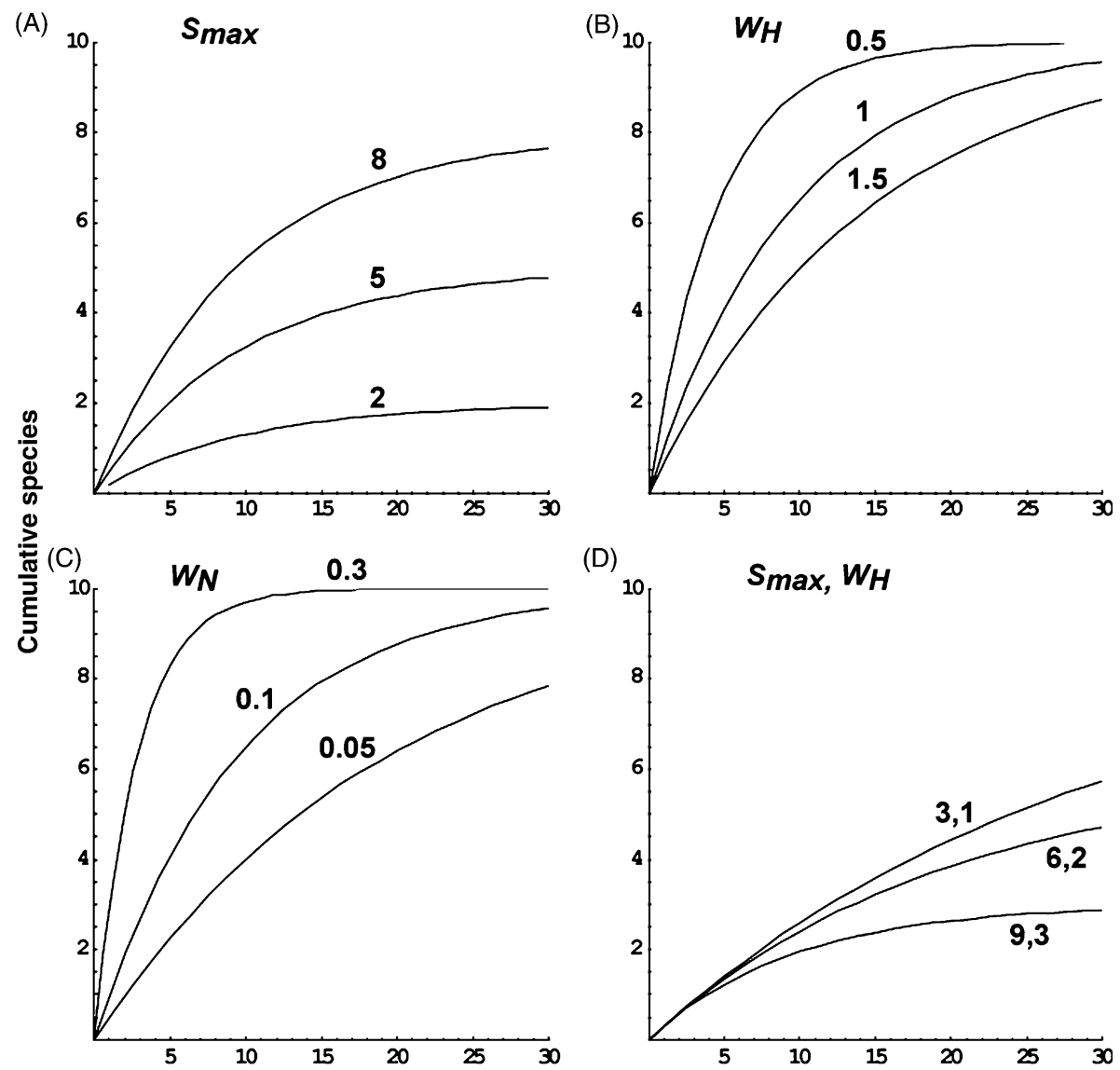

(D)

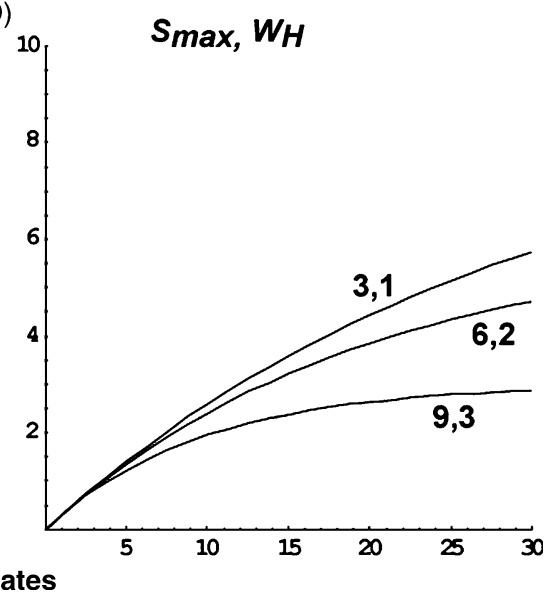

Fig. 2. The effects of $(A)$ the species pool size $\left(S_{\max }\right)$, (B) habitat width $\left(W_{H}\right),(C)$ species' niche width $\left(W_{N}\right)$ and $(D)$ the combined effects of $S_{\max }$ and $\mathrm{W}_{\mathrm{H}}$ in the Abiotic model. The number identifying each curve indicates the value of the parameter being varied. Panel (D) represents the special case where species niche densities across the environmental axis are constant (i.e. the ratio of $S_{\max }$ to $W_{N}$ is remains the same). In each case, the values of the parameters that are not manipulated were as follows: $\mathrm{S}_{\max }=10, \mathrm{~W}_{\mathrm{H}}=1, \mathrm{~W}_{\mathrm{N}}=0.1$.

we expect to find associations between community richness and species turnover through time.

I modeled diversity effects on stability by considering a group of coexisting species drawn from a larger pool. The species pool represents all species that occur in the habitat over a long period. When absent, these species may colonize either by dispersal from other habitats in the region, a dormant pool or seed bank, or remnant populations that have dropped below detection limits but are still capable of increasing in density. Each species $\mathrm{i}$ in the pool has some probability of joining the standing community represented by $\mathrm{c}_{\mathrm{tot}, \mathrm{i}}$ (total colonization rate). When present in the living community, species i's probability of disappearing is given by $\mathrm{e}_{\mathrm{tot}, \mathrm{i}}$ (total extinction rate). These colonization and extinction rates consist of the sum of two components; a species-specific invariant rate $\left(c_{i}\right.$ and $\left.e_{i}\right)$ and the combined effects of all other resident species. Each coexisting species has some average marginal effect on the rate of appearance and disappearance of other species which we call the colonization and extinction factors $\left(c_{f}\right.$ and $\left.e_{f}\right)$. These factors can be intuited as the average marginal effect of each additional community member on other species' likelihoods of joining or departing from the community. Each species' dynamics are described by

$\mathrm{c}_{\mathrm{tot}, \mathrm{i}}=\mathrm{c}_{\mathrm{i}}+\mathrm{c}_{\mathrm{f}} \mathrm{S}$

$\mathrm{e}_{\mathrm{tot}, \mathrm{i}}=\mathrm{e}_{\mathrm{i}}+\mathrm{e}_{\mathrm{f}} \mathrm{S}$

where $S$ is the richness of the extant, living community. Each species has a unique value for $c_{i}$ and $e_{i}$ whereas $c_{f}$ and $e_{f}$ are the same for all species. Each community member can either increase or decrease the likelihood of colonization from the species pool, or the rate of disappearance from the living community. If high diversity repels invasion (Elton 1958), then $c_{f}$ will be negative and species are less likely to join richer communities. If species facilitate colonization through 
mutualisms such as habitat modification (Bruno et al. 2003), then $c_{f}$ is positive. If high diversity increases extinction likelihood by either increasing variance as proposed by May (1974) or reducing mean abundance, then $e_{f}$ is positive. If diversity is a stabilizing force and reduces extinction as suggested by McCann et al. (1998), then $e_{f}$ is negative. I modeled all four cases (positive and negative $\mathrm{e}_{\mathrm{f}}$ and $\mathrm{c}_{\mathrm{f}}$ ), plus non-interactive communities $\left(e_{f}=c_{f}=0\right)$ and examined the effects of adding species to the pool on the rate of compositional turnover in community composition.

I performed numerical simulations for different sized species pools with values for $c_{i}$ and $e_{i}$ drawn from random uniform distributions between 0 and 1 . Values for $c_{f}$ and $e_{f}$ were assigned and the size of the species pool was varied between 5 and 100 species. Each simulation ran for 100 time steps with all species initially absent. Species-time curves were constructed beginning with the tenth time step when richness had equilibrated, and were based on 50 time steps. Although some of the curves reached maxima after 50 time steps (unlike empirical data, which tend to increase indefinitely, Adler et al. 2005), choosing different cutoffs had no effect on the main conclusions. A power function was fit to the species accumulation curve analogous to a species-area curve where $S_{\text {tot }}=\mathrm{aT}^{\mathrm{z}}$. In this function, a is an estimate of instantaneous or daily richness and $\mathrm{z}$ is the rate of species accumulation. (I use a rather than the more typical $\mathrm{c}$ to avoid confusion with the colonization parameter in the model). A high value for $\mathrm{z}$ indicates large changes in species composition between dates (Rosenzweig 1995, Adler et al. 2005, White et al. 2006). We can therefore consider $\mathrm{z}$ to be the inverse of stability. I used the model to ask how a and $\mathrm{z}$ change with the size of the species pool under each hypothesized diversitystability relationship (positive and negative $c_{f}$ and $e_{f}$ ).

Figure 3 shows species accumulation curves as a function of species pool size in each scenario. Fig. 4 summarizes the results in terms of daily richness (a) and turnover $(\mathrm{z})$. The results for the five modeled scenarios are as follows:

1. No interactions. In the non-interactive case, increasing the size of the species pool led to higher a (daily richness) and no changes in turnover (z, Fig. 3A, 4A-B).

2. Diversity reduces colonization. When diversity prevented colonization (negative $\mathrm{c}_{\mathrm{f}}$ ), a increased as the species pool grew, approaching an upper asymptote as it became increasingly difficult to add more species at high richness (Fig. 3B, 4A). Turnover $(\mathrm{z})$ increased with richness in the case of invasion resistance (Fig. 4B). Since species had lower colonization rates into richer communities, new species continued to accumulate over longer periods, leading to greater turnover.
3. Diversity increases colonization. When species facilitated invasion (positive $c_{f}$ ), a increased with the species pool while $\mathrm{z}$ decreased, approaching a minimum (Fig. 3C, 4A-B). In this case, speciestime curves became flatter at higher diversity because more of the species pool was present at each time.

4. Diversity reduces extinction. When diversity was stabilizing (negative $e_{f}$ ), a increased with the size of the species pool while $\mathrm{z}$ decreased below 30 species (Fig. 3D, 4C-D). Low turnover at high diversity occurred for the same reason as in the case of facilitation (Fig. 3C); the species pool was exhausted more quickly as a greater proportion of the species was present at all times.

5. Diversity increases extinction. When diversity was destabilizing (positive $e_{f}$, Fig. 3E, 4C-D), a larger species pool led to higher instantaneous richness (a) but had no effect on turnover $(\mathrm{z})$, the same as in the non-interactive case (Fig. 3A). Each species persisted for a shorter period but new populations appeared at the same rate, leading to no effects on $\mathrm{z}$.

\section{Discussion}

The models implicate two potential mechanisms for the observed pattern of flatter species-time curves with increasing diversity. First, lower turnover through time and greater average diversity can both occur in less variable environments. Habitat conditions that vary over a wider range intersect with fewer species' niches at any moment in time, leading to lower average diversity and steeper species-accumulation curves that increase for longer periods (Fig. 2B). However, this result only applies when the size of the long-term species pool $\left(S_{\max }\right)$ is independent of the degree of abiotic variability. If diversity is driven by the size of the available species pool, then the scaling exponents are equal for all levels of diversity (Fig. 2A). Increasing abiotic variability via $\mathrm{W}_{\mathrm{H}}$ means that the density of species niches along the environmental axis declines as variability increases (Fig. 1), therefore each species is present for a smaller proportion of time (lower $\mathrm{p}_{\mathrm{i}}$ ). If the distribution of species' niches is independent of environmental variability, then species-time curves diverge at long temporal scales (Fig. 2D), the opposite of the observed pattern. In this case, more variable habitats sample the niche space of more species, leading to greater turnover at higher diversity. Thus, the relationships among abiotic variability, diversity and turnover depend on how species niches are distributed along environmental axes and respond to abiotic variability.

The Biotic model shows that convergent species-time curves are consistent only with the cases where diversity reduces extinction probabilities or increases colonization. The model explicitly assumes that diversity is the major 


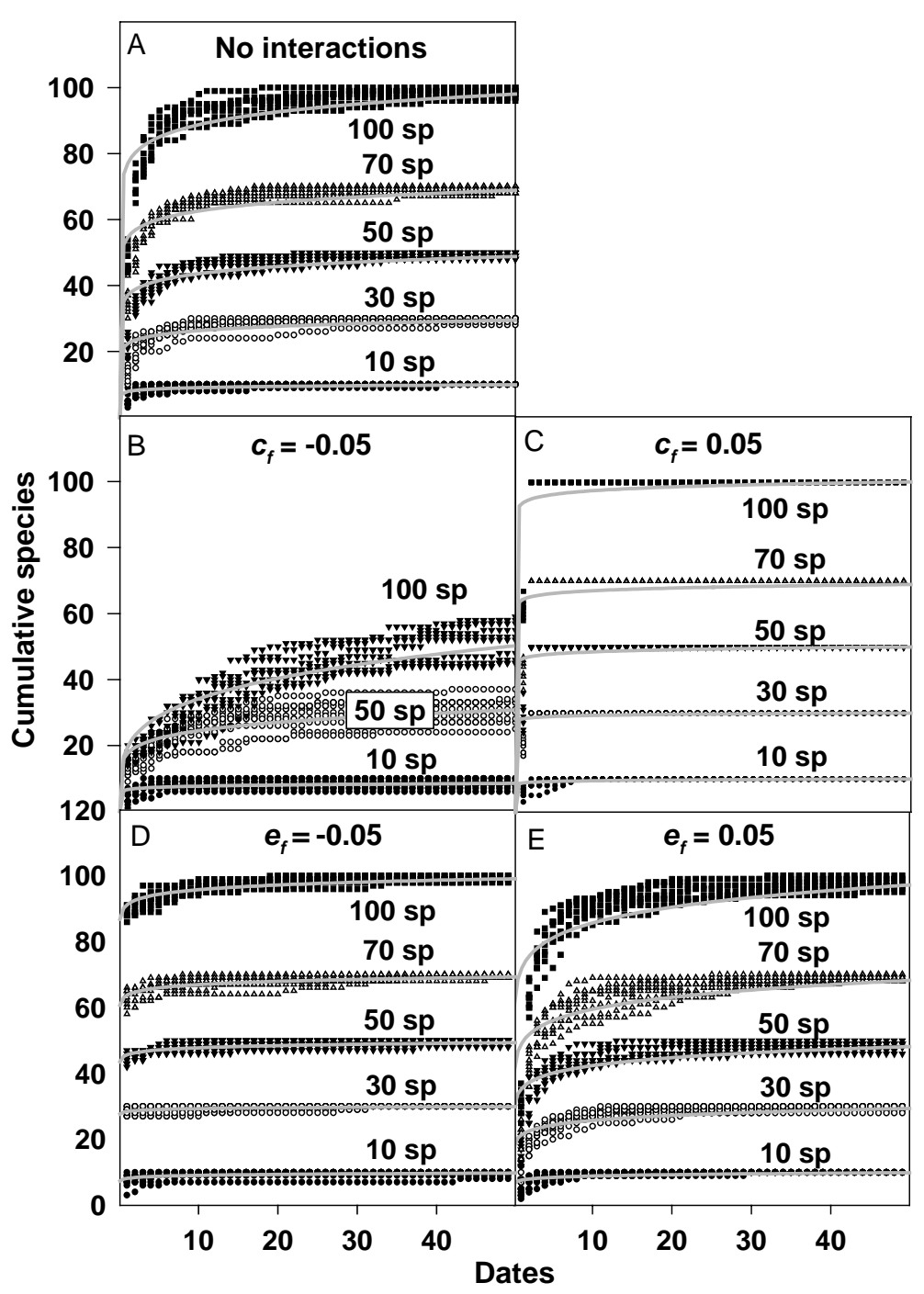

Fig. 3. Species accumulation curves for each of the four modeled scenarios: (A) no interactions $\left(c_{f}=e_{f}=0\right)$, $(B)$ diversity promotes invasion resistance $\left(c_{f}=-0.05, e_{f}=0\right),(C)$ diversity promotes colonization (facilitation, $\left.c_{f}=0.05, e_{f}=0\right),(D)$ diversity reduces extinction $\left(c_{f}=0, e_{f}=-0.05\right)$ and $(E)$ diversity increases extinction $\left(c_{f}=0\right.$, $\left.e_{f}=0.05\right)$. Each set of points shows the results of five simulations with $e_{i}$ and $c_{i}$ drawn from random uniform distributions between 0 and 1 .

driver of extinction and colonization rates in communities. This assumption is the basis of many models of diversity and stability (MacArthur 1955, Elton 1958, May 1974, McCann et al. 1998). The Biotic model with no interactions $\left(c_{f}\right.$ and $\left.e_{f}=0\right)$ is analogous to the Abiotic model where diversity is determined by the size of the available species pool and varies independently of the environment. In both cases, species-time curves are parallel and their slopes are unaffected by diversity. The model suggests that empirical patterns are most consistent with facilitative interactions among species that lead to reduced extinction or enhanced colonization at high diversity. Such interactions can occur due to modification of the physical environment, for instance by "ecosystem engineers", or indirect trophic interactions such as keystone predation (Bruno et al. 2003). Alternatively, high diversity may bound the dynamics of unstable species combinations if weak interactions are prevalent, thereby preventing outbreaks and extinctions (McCann et al. 1998). The model with invasion resistance arising at high diversity predicts diverging species-time curves (Fig. 3C), in contrast with empirical patterns.

The conditions where declining turnover coincides with high diversity in the two models suggest that feedbacks between diversity and colonization and extinction rates (as in the Biotic model) may be more likely explanations for the observed empirical patterns. First, greater habitat variability can promote species 


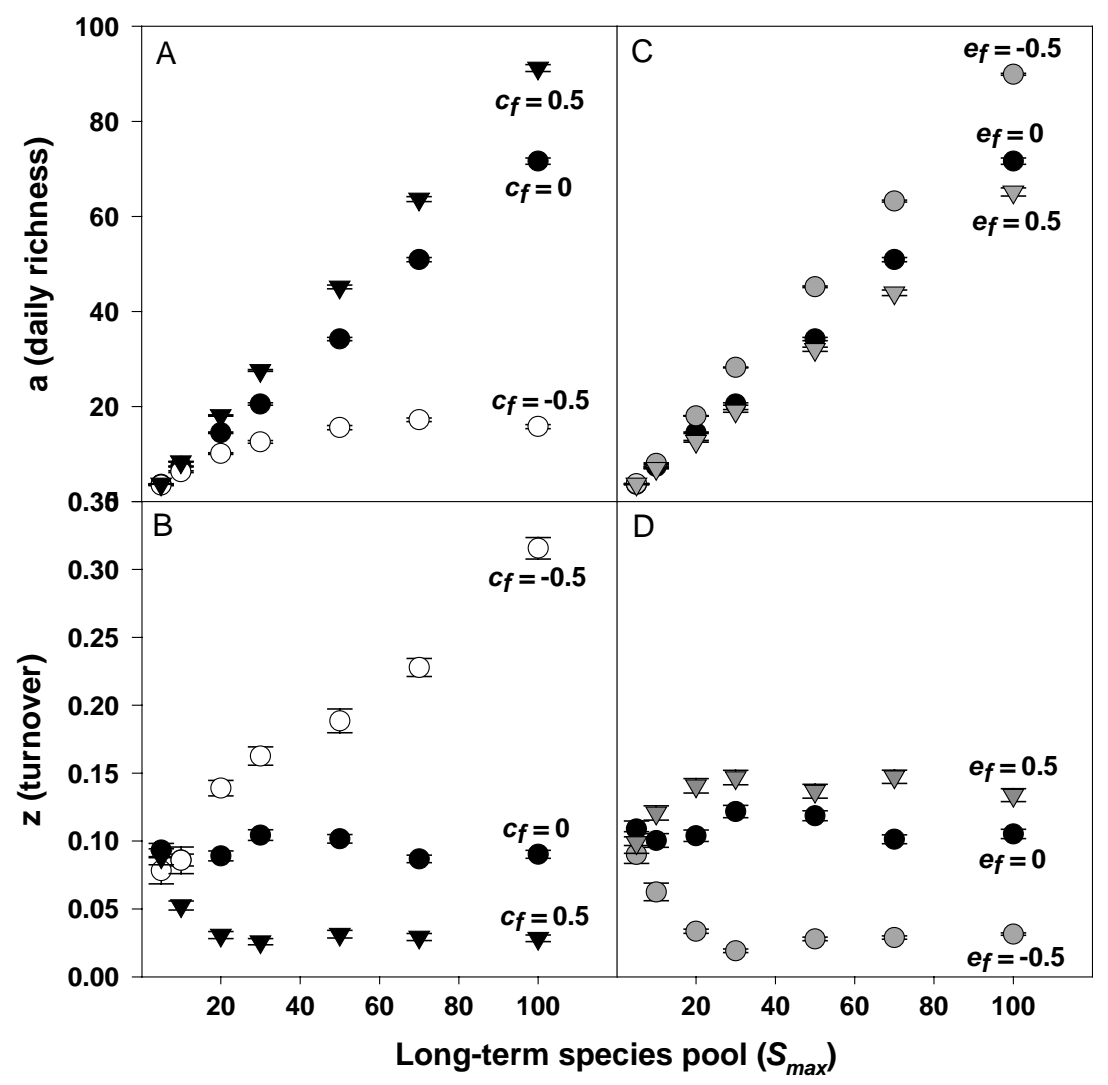

Fig. 4. A summary of the effects of the size of the species pool on (A and C) instantaneous diversity (a) and (B and D) turnover (z) for each of the four modeled cases: no interactions $(\mathbf{O})$, facilitation $\left(\boldsymbol{\nabla}, \mathrm{c}_{\mathrm{f}}=0.05\right)$, invasion resistance $\left(\bigcirc, \mathrm{c}_{\mathrm{f}}=-0.05\right)$, stabilization $\left(O, e_{f}=-0.05\right)$, and destabilization $\left(\nabla, e_{f}=0.05\right)$. The two left panels $(A$ and $B)$ show effects of varying $c_{f}$ and the two right panels $(C$ and $D)$ show effects of $e_{f}$.

diversity through the storage effect, or the ability of species to partition time under changing conditions (Chesson and Warner 1981). Enhanced coexistence through environmental fluctuations has been demonstrated in groups as diverse as lake zooplankton (Caceres 1997) and prairie plants (Adler et al. 2006). These result indicate that temporal variability generally increases the size of the potential species pool. In this situation, species accumulation curves are expected to diverge at long scales. Converging species-time curves occur with increasing abiotic fluctuations only when the long-term species pool is constant across a range of habitat variability (Fig. 2B). Second, a variety of evidence suggests that high diversity may stabilize populations in a community context (McCann 2000). Although diversity may destabilize populations of competitors (May 1974, Ives and Hughes 2002), a variety of empirical studies have shown stabilizing effects of diversity on populations, especially in multi-trophic communities (McCann 2000, Carr et al. 2002, Steiner et al. 2005, 2006). If diversity prevents extinction through stabilization, or promotes colonization through inter-trophic level facilitation, then we expect flatter species-time curves at higher diversity (Fig. 3, 4).

A number of caveats are important to consider when evaluating the simple, heuristic models presented here as they ignore several aspects of biotic interactions and species-environment relationships that are very likely to be important for species turnover. The Biotic model considers only the mean effect of species interactions on colonization and extinction rates, however real communities are structured by a range of competitive, predatory, mutualistic and commensal interactions (Callaway et al. 2002). The variability in the sign of species interactions may be as important as the mean in determining patterns of species turnover. The Abiotic model also considers only environmental variability in time, however spatial variability within habitats may determine how temporal variability affects turnover. For instance, some species may persist under high temporal variability if spatial variability within a patch provides refuges during times when conditions fall outside the species' tolerance range. Spatial and temporal variability may therefore interact in shaping turnover. In addition, temporal variability may 
reduce species' geometric mean fitness, its average population size and potentially the breadth of its environmental tolerance as well. These issues are beyond the scope of the present models, but are likely important for understanding the relationship between diversity and temporal compositional stability. More sophisticated hypotheses about diversity and temporal turnover will likely necessitate the development of more sensitive and informative measures of turnover than the exponent of the species-time curve.

Drawing inferences about biological processes from macroscopic patterns of diversity illustrates the frequent problem in ecology of multiple causality leading to convergent predictions. Patterns of species turnover through time are analogous to species-area and species-abundance distributions in that the shapes of particular functions or the values of statistically derived parameters are often uninformative for distinguishing among competing hypotheses (Williams 1943, McGill 2003). However, the models presented here suggest that the observed negative correlation between the average number of species in a community and the slope of the species-time relationship is consistent with some models of how the environment affects species turnover, and how diversity affects colonization and extinction. High turnover and low diversity (as often observed empirically) occur with greater abiotic variability when the distribution of species niches is independent of the degree of environmental heterogeneity. The Biotic model suggests that any potential diversity effects on population trajectories are generally stabilizing in that they either decrease extinction rates or facilitate colonization. The patterns are inconsistent with neutral effects of diversity on colonization and/or extinction, or biotic resistance to invasion at high diversity. Empirical associations between species turnover and variation in the physical environment may help distinguish between the predictions of the Biotic and Abiotic models. The models provide a processbased interpretation of species-time relations in terms of environmental variability, colonization and extinction.

Acknowledgements - Peter Adler, Alistair Blanchford, Chris Harley, Allyson Longmuir, Russ Markel, Sandra Nicol, Shane Richards, Laura White and Spencer Wood provided helpful comments on the work. Funding was provided by the Natural Sciences and Engineering Research Council of Canada.

\section{References}

Adler, P. B. and Lauenroth, W. K. 2003. The power of time: spatiotemporal scaling of species diversity. - Ecol. Lett. 6: 749-756.

Adler, P. B. et al. 2005. Evidence for a general species-timearea relationship. - Ecology 86: 2032-2039.
Adler, P. B. et al. 2006. Climate variability has a stabilizing effect on the coexistence of prairie grasses. - Proc. Natl Acad. Sci. USA 103: 12793-12798.

Brown, J. H. et al. 2001. Regulation of diversity: maintenance of species richness in changing environments. - Oecologia 126: 321-332.

Bruno, J. F. et al. 2003. Inclusion of facilitation into ecological theory. - Trends Ecol. Evol. 18: 119-125.

Burke, M. J. W. and Grime, J. P. 1996. An experimental study of plant community invasibility. - Ecology 77: 776-790.

Caceres, C. E. 1997. Temporal variation, dormancy, and coexistence: a field test of the storage effect. - Proc. Natl Acad. Sci. USA 94: 9171-9175.

Callaway, R. M. et al. 2002. Positive interactions among alpine plants increase with stress. - Nature 417: 844848.

Carr, M. H. et al. 2002. Biodiversity, population regulation, and the stability of coral-reef fish communities. - Proc. Natl Acad. Sci. USA 99: 11241-11245.

Chesson, P. L. and Warner, R. R. 1981. Environmental variability promotes coexistence in lottery competitive systems. - Am. Nat. 117: 923-943.

Chesson, P. and Huntly, N. 1997. The roles of harsh and fluctuating conditions in the dynamics of ecological communities. - Am. Nat. 150: 519-553.

Connor, E. F. and McCoy, E. D. 1979. Statistics and biology of the species-area relationship. - Am. Nat. 113: 791833.

Crawley, M. J. and Harral, J. E. 2001. Scale dependence in plant biodiversity. - Science 291: 864-868.

Davis, M. A. et al. 2000. Fluctuating resources in plant communities: a general theory of invasibility. - J. Ecol. 88: $528-534$.

Elton, C. S. 1958. The ecology of invasions by animals and plants. - Metheun.

Gotelli, N. J. and Colwell, R. K. 2001. Quantifying biodiversity: procedures and pitfalls in the measurement and comparison of species richness. - Ecol. Lett. 4: 379391.

Green, J. L. et al. 2004. Spatial scaling of microbial eukaryote diversity. - Nature 432: 747-750.

Hanski, I. et al. 1996. Minimum viable metapopulation size. - Am. Nat. 147: 527-541.

Harte, J. et al. 1999. Self-similarity in the distribution and abundance of species. - Science 284: 334-336.

He, F. L. and Legendre, P. 1996. On species-area relations. - Am. Nat. 148: 719-737.

He, F. L. and Legendre, P. 2002. Species diversity patterns derived from species-area models. - Ecology 83: 11851198.

Hillebrand, H. 2004. On the generality of the latitudinal diversity gradient. - Am. Nat. 163: 192-211.

Horner-Devine, M. C. et al. 2004. A taxa-area reltionship for bacteria. - Nature 432.

Hubbell, S. P. 2001. The unified neutral theory of biodiversity and biogeography. - Princeton Univ. Press.

Ives, A. R. and Hughes, J. B. 2002. General relationships between species diversity and stability in competitive systems. - Am. Nat. 159: 388-395. 
Koleff, P. et al. 2003. Are there latitudinal gradients in species turnover? - Global Ecol. Biogeogr. 12: 483-498.

Levine, J. M. and D’Antonio, C. M. 1999. Elton revisited: a review of evidence linking diversity and invasibility. - Oikos 87: 15-26.

MacArthur, R. H. 1955. Fluctuations of animal populations and a measure of community stability. - Ecology 36: 533-536.

MacArthur, R. H. and Wilson, E. O. 1967. The theory of island biogeography. - Princeton Univ. Press.

May, R. M. 1974. Stability and complexity in model ecosystems. - Princeton Univ. Press.

McCann, K. S. 2000. The diversity-stability debate. - Nature 405: 228-233.

McCann, K. et al. 1998. Weak trophic interactions and the balance of nature. - Nature 395: 794-798.

McGill, B. 2003. Strong and weak tests of macroecological theory. - Oikos 102: 679-685.

Pimm, S. L. et al. 1988. On the risk of extinction. - Am. Nat. 132: $757-785$.

Preston, F. W. 1960. Time and space and the variation of species. - Ecology 41: 611-627.

Rosenzweig, M. L. 1995. Species diversity in space and time. - Cambridge Univ. Press.

Schoener, T. W. and Spiller, D. A. 2006. Nonsynchronous recovery of community characteristics in island spiders after a catastrophic hurricane. - Proc. Natl Acad. Sci. USA 103: 2220-2225.

Seabloom, E. W. et al. 2002. Extinction rates under nonrandom patterns of habitat loss. - Proc. Natl Acad. Sci. USA 99: 11229-11234.

Shmida, A. and Wilson, M. V. 1985. Biological determinants of species diversity. - J. Biogeogr. 12: 1-20.

Shurin, J. B. 2000. Dispersal limitation, invasion resistance, and the structure of pond zooplankton communities. - Ecology 81: 3074-3086.

Shurin, J. B. et al. 2007. Diversity-stability relationship varies with latitude in zooplankton. - Ecol. Lett. 10: 127-134.

Steiner, C. F. et al. 2005. The influence of consumer diversity and indirect facilitation on trophic level biomass and stability. - Oikos 110: 556-566.

Steiner, C. F. et al. 2006. Population and community resilience in multitrophic communities. - Ecology 87: 996-1007.

Sugihara, G. 1980. Minimal community structure-an explanation of species abundance patterns. - Am. Nat. 116: $770-787$.

White, E. P. 2004. Two-phase species-time relationships in North American land birds. - Ecol. Lett. 7: 329-336.

White, E. P. et al. 2006. A comparison of the species-time relationship across ecosystems and taxonomic groups. - Oikos 112: 185-195.

Williams, C. B. 1943. Area and number of species. - Nature 152: $264-267$.

\section{Appendix 1}

Here I show that relaxing the assumptions of equivalent niche widths among species and a uniformly distributed and temporally uncorrelated environment do not affect the main conclusions of the model. Figure A1 shows species-time curves from simulations of the Abiotic model where the value of the environmental axis is drawn randomly from a normal distribution and the position and widths of species' niches are from a uniform distribution. The top panels show the effects of increasing $S_{\max }$, the size of the species pool (Fig. A1A), and the bottom shows the effects of the standard deviation of the environmental axis (Fig. A1B). The qualitative patterns are the same as in the basic model with equivalent niche widths and a uniform probability of encountering environmental states - increasing $S_{\max }$ increases the intercept but has no effect on the slope of the species-time curves, while increasing variation in the abiotic environment causes the curves to converge at long time scales. I also simulated situations where environmental variation was temporally autocorrelated (not shown). Autocorrelation was introduced by adding a random increment to the value of the environment from the previous time step. The increment was drawn from a normal distribution with a mean of 0 and standard deviation of 0.5 . The qualitative patterns were also the same in a temporally autocorrelated environment.

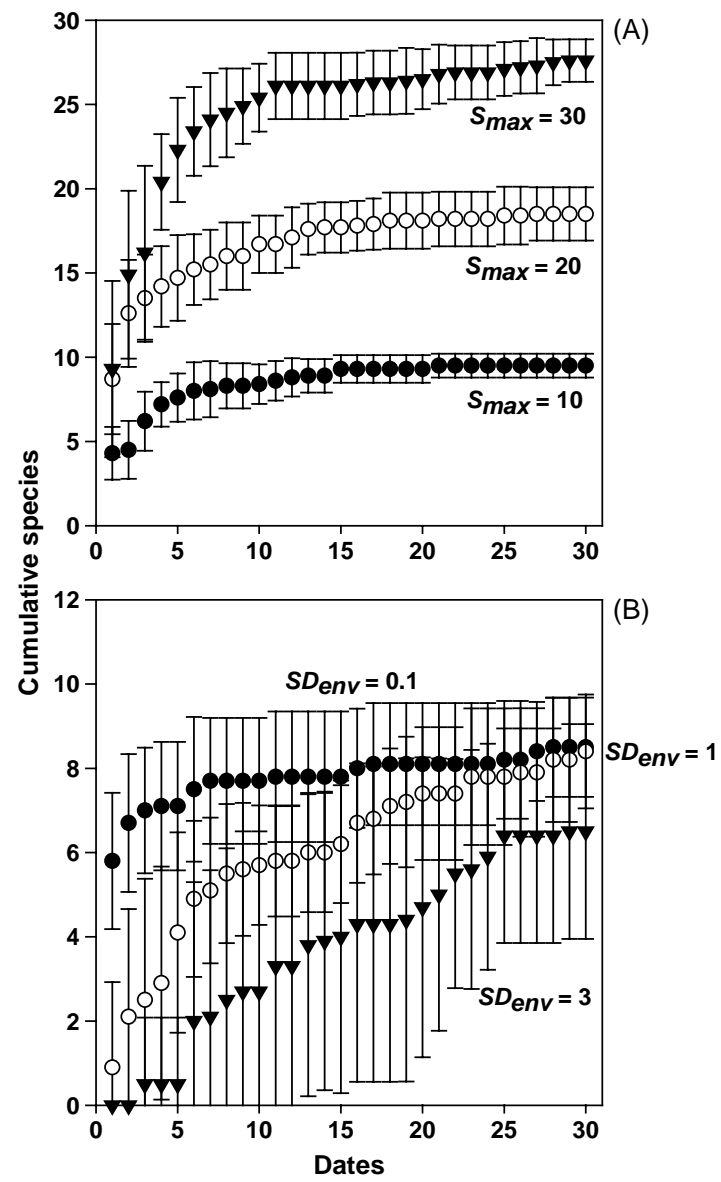

